\title{
El exilio de Simón Bolívar
}

\author{
Dr. Adalberto Santana-Hernández \\ Investigador \\ Centro de Investigaciones sobre América Latina y el Caribe (CIALC) \\ Universidad Nacional Autónoma de México
}

\section{Resumen}

El exilio marca el derrotero de diversos momentos políticos en la mayoría de los países iberoamericanos. A lo largo de distintas etapas de la historia de América Latina y el Caribe, el exilio ha sido una condición y una política en la que el sujeto que lo vive ha tenido que abandonar su país para radicarse en otro; desterrado. En este trabajo abordaremos e identificaremos el exilio político del prócer latinoamericano Simón Bolívar. Analizaremos su actuación en Curazao, Jamaica y Haití.

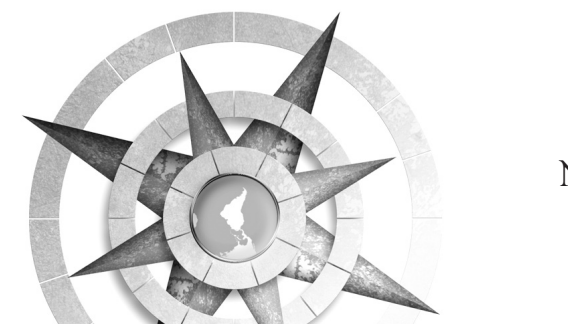

Keywords: Latin American exile; Simón Bolivar, Latin American History, Temas de Nuestra América, Exile Research Group

Palabras clave: exilio latinoamericano, Simón Bolivar, historia latinoamericana, Temas de Nuestra América, Cátedra del Exilio
Abstract
Exile marks the course of various politi- cal moments mostly in all Latin Ame- rican countries. Throughout different stages of the Latin American and $\mathrm{Ca}$ - ribbean history, exile has been a condi- tion and a policy in which the subject banished it has had to leave his coun- try to settle in another in this paper we discuss and identify the political exile of the Latin American forefather, Simón Bo- livar. We analyze his actions in Curaçao, Jamaica and Haiti. 


\section{Introducción}

El fenómeno del exilio ha sido una constante en la historia política y cultural en Iberoamérica. El destierro de diversos actores y grupos ha marcado el derrotero de los procesos políticos en la mayoría de los países latinoamericanos. Su dinámica ha sido un punto neurálgico que tuvo y tiene una incidencia fundamental en el devenir de los procesos históricos, políticos y culturales latinoamericanos. El siglo XIX fue signado por los movimientos independentistas y el surgimiento de nuevos Estados nacionales, el siglo XX por sus conflictos internos y externos (dictaduras, guerras y revoluciones); finalmente, en el inicio del siglo XXI, el fenómeno de los desplazamientos forzados a causa de la violencia política, criminal y los llamados golpes suaves, han generado en la región nuevos elementos del exilio.

Así, podemos reconocer que el exilio marca el derrotero de diversos momentos políticos en la mayoría de los países iberoamericanos; es un punto de inflexión en la biografía de personas que a lo en la historia regional han figurado como un punto de referencia obligada. A lo largo de distintas etapas de la historia de América Latina y el Caribe, el exilio ha sido una condición y una política en la que el sujeto que lo vive ha tenido que abandonar su país para radicarse en otro; desterrado.

Esa situación fue casi permanente durante el proceso histórico de los movimientos políticos latinoamericanos ubicados en la oposición política contraria a los grupos que detenta el poder. Así, actores ideas de corte democrático, progresista o revolucionario, han vivido el destierro y el exilio, aunque también en determinado momento lo han padecido otros de corte conservador o dictatorial, quienes, tras perder el poder, han recurrido al exilio, fenómeno político latinoamericano que se desarrolló durante buena parte de los siglos XIX y XX.

Los exilios de los grandes próceres de las luchas de independencia latinoamericanas son los que han tenido una amplia repercusión; como ejemplos, podemos citar los casos de: Simón Bolívar en Curazao, Jamaica y Haití; Francisco Morazán en Panamá y Perú; Benito Juárez en La Habana y Nueva Orleans; y José Martí en México, Guatemala y Estados Unidos de América.

De igual manera, en determinados conflictos políticos del siglo XIX, encontramos casos de destierro y exilio, como el de la Guerra de los Diez Años en Cuba

1 Cfr. Adalberto Santana, "Exilio y antiimperialismo latinoamericano", en Cuadernos Americanos, núm. 152, abril-junio de 2015, pp. 41-52. 
(1868-1878), acontecimiento político que generó un éxodo masivo.

En los inicios del siglo XX, recordemos que la Revolución Mexicana y el conflicto armado que se dio entre 1910 y 1920, produjeron el destierro y el exilio de importantes dirigentes y actores de distintos grupos, especialmente hacia Cuba, Estados Unidos y Europa.

Por otra parte, uno de los fenómenos históricos que tuvo un impacto profundo en la sociedad latinoamericana fue el arribo de desplazados durante la Primera Guerra Mundial, con el derrumbe del Imperio Otomano y el de los refugiados españoles, como consecuencia de la Guerra Civil (1936-1939), que vino aparejada con la presencia de otros tantos refugiados antifascistas procedentes del centro de Europa, muchos de ellos de origen judío, polaco, ruso y alemán, entre otros.

Asimismo, figuró en el siglo $\mathrm{XX}$ el exilio de destacados personajes políticos de diversas nacionalidades como Augusto C. Sandino, Raúl Haya de la Torre, León Trotsky, Rómulo Gallegos, José Figueres, Jacobo Arbenz Guzmán, Juan Bosch, Fidel y Raúl Castro, Omar Torrijos y Daniel Ortega, por mencionar algunos de los más relevantes.
Estos últimos exiliados en gran medida vivieron el destierro como consecuencia de las políticas represivas de las dictaduras centroamericanas y caribeñas, entre ellas, podemos mencionar las de la dinastía Somoza en Nicaragua, de Rafael Leonidas Trujillo, en República Dominicana y de Fulgencio Batista en Cuba; así como las sudamericanas, que produjeron el terror, como las ejercidas por los generales Augusto Pinochet y Jorge Rafael Videla en Chile y Argentina, respectivamente. También las implantadas en Brasil, Bolivia, Paraguay y Uruguay; o bien, las dictaduras militares y las guerras que se sucedieron en Centroamérica durante el siglo XX, las cuales generaron un flujo de miles de ciudadanos que se vieron obligados a emigrar forzadamente a otros países de la región o fuera de ella. La lista puede, sin duda, ser tremendamente numerosa. Pero en nuestros días de inicios del siglo XXI, predomina un nuevo exilio agravado por condiciones de exclusión y violencia. Pensemos que el estudio del exilio ha requerido hacer y

[...] una lectura matizada del contexto y la historia, porque evolucionó y cambió de carácter a lo largo de los siglos XIX y XX. El exilio es dinámico, con eje en la acción política, y con una evolución paralela a los procesos de

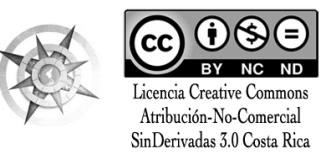


institucionalización y desinstitucionalización políticas, así como a reformulación de las reglas políticas básicas. A la vez, las dinámicas del exilio recurrente han sido componentes principales de las democracias limitadas o excluyentes en América Latina. ${ }^{2}$

De esta manera, en el escenario latinoamericano, e incluso mundial, podemos encontrar diversas expresiones del exilio, de la migración forzada o de desplazamientos forzados muy recurrentes en México, España, Centroamérica y Sudamérica a lo largo de los siglos XIX, XX, e incluso en los comienzos del siglo XXI. ${ }^{3}$

2 Sznajder Mario y Luis Roniger, La política del destierro y el exilio en América Latina, México, FCE, 2009, p. 27.

3 Se habla de una esclavitud moderna que genera ganancias superiores a los 150 mil millones de dólares, que en buena medida se encuentra no visible, según denunció la Organización No Gubernamental A21. La cantidad de personas que viven en esa condición en el mundo, según el Índice Global de Esclavitud (IGE), difundido por la Fundación Walk Free, se estima en 45 millones 800 mil personas ("información generada en más de 40 mil entrevistas en 52 países”). Según el IGE, los sectores más afectados por ese tipo de esclavitud son: "niños, hombre y mujeres, que son obligados al trabajo sin remuneración, explotación sexual e incluso mendigar. En lo que respecta al trabajo forzado, se tiene identificado que son mujeres las más afectadas, al ser obligadas a laborar en industrias como la minera, agrícola, de construcción o en labores domésticas. El problema está focalizado en América Central, del Norte, y en el Caribe". Incluso se sostiene que en México alcanza el 70 por ciento de los casos de esclavitud moderna, relacionados con grupos del
En este tenor de ideas, se afirma que las dinámicas del exilio en Iberoamérica han sido una constante en la historia de esta región. Asimismo han marcado de manera muy significativa las identidades de diversos países, reflejando mucho de la cultura y el imaginario colectivo de las sociedades. La consolidación de los Estados nacionales no puede entenderse sin el exilio político, que fue utilizado como mecanismo de exclusión por parte de gobiernos que buscaban afianzar su radio de acción. El fenómeno se compuso primeramente entre los círculos políticos y económicos que se disputaban el poder, y posteriormente se fue extendiendo a los grupos de oposición o disidentes a los regímenes autoritarios, como los trabajadores y sindicatos, profesionistas, estudiantes, intelectuales, dirigentes políticos o cualquier otra persona o grupo inmiscuido en las cuestiones politicas de sus países, contrarios a los intereses de los grupos en el poder gubernamental.

En la misma historia de la región, en los siglos pasados, el desplazamiento forzado por motivos políticos ha sido producto de las guerras (tanto aquellas que buscaban la independencia o en las que se defendía la soberanía nacional).

crimen organizado. Así, se encuentra un escenario donde a los grupos de mayor vulnerabilidad se les identifica con "las mujeres y los niños, las poblaciones indigenas, las personas con discapacidad, los migrantes y las personas de la comunidad gay”. Crónica, México, 25 de septiembre de 2016, p. 12 . 
Así, por ejemplo, la Guerra de los Diez Años en Cuba produjo un éxodo masivo en diversos países que ya habían logrado su independencia y estaban en un proceso de profundas reformas liberales, como México, Guatemala, Honduras y Costa Rica.

En este contexto destaca el exilio político de grandes actores que han impactado por su obra y acciones en la historia regional y mundial; en este artículo abordaremos e identificaremos el exilio político de Simón Bolívar, prócer latinoamericano que ha contado con una mayor visibilidad y repercusión; veremos su actuación en Curazao, Jamaica y Haití.

\section{El exilio de Simón Bolívar}

Uno de los personajes más importantes en la historia política de los países latinoamericanos es, sin lugar a dudas, Simón José Antonio de la Santísima Trinidad Bolívar y Palacios de Aguirre Ponte-Andrade y Blanco, Simón Bolívar, el gran Libertador.

El más grande libertador de nuestra América nació el 24 de julio de 1783, y murió el 17 de diciembre de 1830, en la Hacienda de San Pedro Alejandrino, muy cercana a la caribeña ciudad de Santa Martha, Colombia. El fallecimiento del Libertador es tratado por el escritor Gabriel García Márquez en su célebre novela El general en su laberinto. Podemos afirmar con toda justicia que la presencia e imagen de Simón Bolívar lo hacen, sin lugar a dudas, el personaje histórico latinoamericano más homenajeado en todo el mundo. Su nombre figura de manera destacada en todas las naciones de Latinoamérica; y de igual forma, se le reconoce en varios espacios púbicos. Su veneración está plasmada en numerosas calles, avenidas bibliotecas, auditorios y teatros que llevan su nombre, como en Bolivia y la República Bolivariana de Venezuela, incluso diversos organismos regionales ostentan su nombre, como la Alianza Bolivariana para los Pueblos de Nuestra América (ALBA), por ejemplo.

Simón Bolívar es un personaje representado en estatuas, bustos, murales y pinturas de famosos artistas, así como en poesías, novelas, cuentos, obras de teatro y otros trabajos literarios de reconocidos autores, entre ellos Pablo Neruda, quien dedicó "Un canto para Bolívar". También lo encontramos en diversos ensayos. Al respecto, José Martí expresa: “¡Pero así está Bolívar en el cielo de América, vigilante y ceñudo, sentado aún en la roca de crear, con el inca al lado y el haz de banderas a los pies; así está él calzadas aún las botas de campaña, porque lo que él no dejó hecho, 
sin hacer está hasta hoy: porque Bolivar tiene que hacer en América todavía!". 4

Podemos afirmar que los pueblos y los gobiernos de nuestra América han identificado en Bolívar al precursor de las ideas y proyectos de integración regional. Pensemos que el ideario latinoamericanista que urge desarrollar en este siglo XXI, en el cual se han dado importantes pasos para llegar a ese permanente anhelo regional propuesto desde hace doscientos años, tiene como figura egregia al gran Libertador. Este personaje histórico, siendo muy joven, se embarcó en el puerto de La Guaira, en el navío San Ildefonso con destino a España, y siguiendo una ruta que requería hacer escala en La Habana. Sin embargo, en aquel momento ésta se hallaba ocupada por los ingleses, por lo que hace que finalmente navegue hacia Veracruz, a donde llega en febrero de 1799; así, Bolívar aprovecha la ocasión para visitar la Ciudad de México.5 En ese entonces el joven caraqueño contaba con 15 años de edad. Desde aquel año de 1799 hasta el 1802, radicó en

4 José Martí, "Discurso pronunciado en la velada de la Sociedad Literaria Hispanoamericana en honor de Simón Bolívar el 28 de octubre de 1893", en Obras completas, t. 8, La Habana, Editorial de Ciencias Sociales, 1975, p. 243.

5 Cfr. Rafael Heliodoro Valle (compi., pról. y notas), Bolivar en México, México, SER-Acervo Histórico Diplomático, 1993; y Gustavo Vargas Martínez, Presencia de Bolivar en la cultura mexicana, México, UNAM, 2005.
España y recorrió varios lugares de Europa. Después de la muerte de su esposa María Teresa del Toro y Alayza -enero de 1803- en Caracas, viajó nuevamente a Europa. Allá presenció, en 1804, la coronación de Napoleón en Saint-Cloud. Ese mismo año en que se proclamó la independencia de Haití respecto de Francia. Cuando viajaba por Italia, con la su maestro Simón Rodríguez y su amigo Fernando Toro, el 15 de agosto de 1805 hizo en el Monte Sacro de Roma el siguiente juramento: "iJuro delante de usted; juro por el Dios de mis padres; juro por ellos; juro por mi honor; juro por la patria, que no daré descanso a mi brazo, ni reposo a mi alma, hasta que haya roto las cadenas que nos oprimen por voluntad del poder español!”.

En un segundo viaje, cinco años después, recorrió Europa y entró en contacto con las ideas de importantes filósofos, como John Locke, Rousseau, Diderot, Voltaire y Montesquieu. Además, se relacionó con las ideas ilustradas que se gestaron en este continente durante el siglo XIX y que culminaron con las ideas independentistas de las colonias americanas. Es en esta época cuando Bolívar decidió emprender la lucha por la unificación de América.

6 Simón Bolívar, "Juramento de Roma", en Por la libertad de mi patria. Discursos, cartas y decretos, México, CONACULTA, 2015, p. 8. 
En 1806, mientras Bolívar se encontraba en Francia, otro exiliado hispanoamericano también nacido en Caracas, de nombre Francisco de Miranda, organiza dos expediciones para independizar a Venezuela, pero fracasaron. Un año más tarde en 1807, Bolívar desembarcó en Charleston (Carolina del Sur, Estados Unidos) y visitó las ciudades de Filadelfia, Nueva York y Boston. De regreso a Caracas, a mediados de aquel año precisamente donde nació, Simón Bolívar organizó una serie de reuniones en la "Cuadra Bolívar" para conversar y reflexionar sobre literatura, oír música y conspirar contra las autoridades coloniales. También ese año se introdujo la imprenta en la Capitanía General de Venezuela; el 28 de octubre fue publicado el primer periódico, la Gazeta de Caracas, cuyo redactor fue otro gran maestro de Bolívar el señor Andrés Bello.

En esos instantes el continente dominado por las fuerzas españolas, comenzó a vivir una fuerte ebullición independentista: en 1809, en Quito, inició el proceso emancipador -con la formación de la Junta Gubernativa, que más tarde -10 de agosto- va a ser disuelta por las fuerzas realistas. $\mathrm{Al}$ año siguiente esa ebullición independentista se ahonda en varias regiones de los territorios coloniales españoles de América. En el virreinato del Río de la Plata se inició la Revolución de Mayo, que triunfó en Buenos Aires. En Bogotá, capital del Virreinato de Nueva Granada arranca la lucha por la independencia con la prisión del virrey Amat (20 de agosto). En la Nueva España comenzó la revolución de independencia encabezada por Miguel Hidalgo y Costilla, ex rector del Colegio de San Nicolás. En tanto que en España, al mismo tiempo comienzan las Cortes de Cádiz.

Mientras el coronel Simón Bolívar es nombrado comisionado ante el Gobierno Británico, por la Junta de Caracas, lo acompañó su maestro Andrés Bello, entre otros. En Londres, estableció contacto con José de Miranda el más destacado de entonces, exiliado hispanoamericano independentista, quien arribó a Venezuela al final de diciembre de 1810, otorgándole la Junta de Gobierno de Caracas el grado de general. En ese mismo contexto el 14 de mayo de 1811 se constituyó en Asunción la Junta Gubernativa del Paraguay, bajo la conducción del doctor Gaspar Rodríguez de Francia. En tanto que en la noche del 3 y la madrugada del 4 de julio, Bolívar dirigió unas palabras en la Sociedad Patriótica, abogando por la inmediata proclamación de la Independencia, lo cual realizó el día el 5 el Congreso Constituyente de Venezuela (fue la primera proclamación de 
independencia de Hispanoamérica). Inició un año terrorífico en la historia, al producirse en marzo de 1812 el terremoto que casi destruyó completamente las ciudades de Caracas, La Guaira, Barquisimeto, Mérida y sus alrededores.

Con ese escenario, Bolivar tomó el mando de Puerto Cabello como comandante político y militar. Mantuvo la resistencia frente a la sublevación en el momento en que José de Miranda capitulaba ante el jefe realista Domingo de Monteverde. Tras ofrecer resistencia ante a las fuerzas realistas, Bolívar tuvo que exiliarse en la isla de Curazao (entre el 1 de septiembre y mediados de octubre). En este periodo concluyó lo que se denominó la Primera República de Venezuela. Éste fue el primer destierro de Bolivar, que duró "cerca de cincuenta días que pasó en Curazao".? En esa isla, frente a las costas venezolanas y en posesión de Holanda, Bolívar tiene una permaneció de casi dos meses. Ahí el joven Bolívar de veintinueve años de edad:

[...] logró curar, gracias a Mordechay Ricardo y sus amigos curazoleños, su angustia, su interior enfermo, recobrar nuevas fuerzas y ponerse de nuevo, con hondos bríos, en aquello a lo cual había jurado dedicar su vida: la indepen-

7 Roberto J. Lovera de-Sola, Curazao, escala en el primer destierro del Libertador, Caracas, Monte Avila Editores, 1992, p. 38. dencia de Suramérica. De allí que fue en la ínsula holandesa donde redactó la primera versión de su primer documento público de significación, el que sería conocido como el Manifiesto de Cartage$\mathrm{na}^{8}$, ya que fue publicado en esa ciudad de la Nueva Granada?

Bolívar llegó el 2 de noviembre a Cartagena de Indias, que se encontraba como un territorio independiente de la hegemonía del colonialismo español que imperaba en el continente hispanoamericano. Para los inicios de 1813 (enero y febrero) Bolívar organizó su ejército en las poblaciones de Ocaña y Mompox y consiguió liberar a Cúcuta. En el fragor de esa lucha independentista, recibió la autorización del presidente de la Confederación de Nueva Granada, Camilo Torres, para incursionar en la Capitanía General de Venezuela. Así, al entrar a la ciudad de Mérida el 23 de mayo de aquel año, fue aclamado como el Libertador. Como se sabe, en mayo de ese año de 1813 emprendió la "Campaña Admirable", periodo que se caracterizó por la reconquista de los territorios occidentales de la Capitanía General de Venezuela.

8 En Simón Bolivar, Escritos del Libertador, t. IV, Caracas, Sociedad Bolivariana de Venezuela, 1964, pp. 116-127, ibid., p. 41.

9 R. J. Lovera de-Sola, op. cit., p. 41. 
Con un ejército numeroso reunió las condiciones para lanzar una nueva ofensiva contra los españoles y canarios, tal como lo manifestó en su "Decreto de Guerra a Muerte" del 15 de junio. Es la ya mencionada Campaña Admirable. En poco tiempo pudo llegar a Caracas; el 6 de agosto de ese año, empezó la denominada Segunda República. En Puerto Cabello, las fuerzas realistas encabezadas por Monteverde realizaron un tratado y el canje de prisioneros. En esa coyuntura, Bolívar y las fuerzas independentistas tuvieron que hacer frente a dos bloques militares: el de las tropas realistas españolas y el que encabezaba el caudillo de sectores depauperados como José Tomás Boves y otros jefes que se despliegan en la región costera central del territorio venezolano. El12 de octubre, Paraguay, bajo la conducción del doctor Gaspar Rodríguez de Francia, proclamó su independencia.

Frente al terror de las fuerzas realistas, los primeros días de junio de 1814 gran parte de la población salió de Caracas, emprendiendo un éxodo hacia Oriente. Para mediados de ese mes, la lucha emancipadora encabezada por Bolívar sufre una dura derrota. Tras las batalla de La Puerta, se presentó en el Congreso de Nueva Granada para explicar el desarrollo de la guerra de independencia. Finalizó así el periodo de la lucha independentista en la Capitanía General de
Venezuela, llamado, como hemos señalado, la Segunda República. Ante esa situación Bolívar, se presentó al el gobierno de Nueva Granada, el cual ascendió a general de división, encargándole la tarea de someter al Estado de Cundinamarca a la Unión Granadina.

En el año siguiente, el 23 de enero de 1815, Bolívar es nombrado capitán general de la Confederación de la Nueva Granada. Sale de Bogotá rumbo a Cartagena para tratar de volver a encabezar la lucha por la independencia de Venezuela. El gobernador del Estado de Cartagena le negó el apoyo en armas y pertrechos para continuar su lucha, por la que se vio obligado a separarse para no dividirlo y a exiliarse. "El Congreso aceptó su renuncia y Bolívar el 9 de mayo de 1815 dejó Nueva Granada, embarcando rumbo a Jamaica. En la travesía pudo ver desde la cubierta de su barco el bosque de mastíles de la armada española que se aproximaba a las costas de su sufrida patria". ${ }^{10}$

Finalmente arribó a Kingston, Jamaica, el 14 de mayo de aquel año. Meses después de estar en la colonia británica, el 6 de septiembre redactó la llamada "Carta de Jamaica". ${ }^{11}$ Su llegada a

10 I. Lavretski, Simón Bolívar, Moscú, Editorial Progreso, 1982, p. 63.

11 Cfr. "Contestación de un Americano Meridional a un caballero de esta Isla (Henry Cullen)", en 
Jamaica, tiene varias explicaciones. Al respecto, se anota que "[...] Jamaica era una de las más activas colonias inglesas del Nuevo Mundo, Gobernada por un personaje influyente, el Duque de Manchester, habitada por gentes cultas y atentas a los movimientos de la política y fortalecida por un próspero comercio exterior, su situación en el mar Caribe la convertía en un centro vital de los intereses británicos en esta parte del globo". ${ }^{2}$

De igual manera, Jamaica fue un punto necesario del exilio, ya que ofrecía una serie de condiciones en aquel momento político de la lucha por la independencia hispanoamericana. El imperio español se encontraba en una profunda crisis, y sus órganos de persecución contra los independentistas se hacían sentir con toda su fuerza. Por ejemplo, Francisco de Miranda, precursor de la independencia

Simón Bolívar, Doctrina del Libertador, Caracas, Biblioteca Ayacucho, 1976, pp. 55-75. Se apunta que: 'Lo curioso en la 'biografía' de la Carta de Jamaica sería el hecho de que su destinatario indeterminado, sólo mencionado como 'Caballero de la Isla', hubiese permanecido durante largo tiempo en el anonimato. Al menos, hubo cierta duda respecto a la identidad del comerciante inglés $\mathrm{Mr}$. Henrry Cullen, residente de Falmouth, puerto en el noroeste de la isla, ubicado en diagonal con la capital Kingston, situada en el opuesto sureste", Kaldone Nweihed, "Palabras preliminares", en Reinaldo Rojas, Bolivar y la Carta de Jamaica, Barquisimeto, 2015, Ediciones Moon, p. 21.

12 Ramón Díaz Sánchez, "Proyecciones históricas de la Carta de Jamaica” en Boletín de la Academia Nacional de Historia, Caracas, t. XLVIII, núm. 191, p. 355, citado en R. Rojas, op. cit., p. 36. americana que vivió largos años en el exilio, especialmente bajo la protección inglesa, finalmente fue recluido en la prisión de La Carraca (San Fernando, Cádiz, España), en donde fallece el 14 de julio de 1816. Su exilio en Jamaica que se prolongó durante siete meses, le permitió a Bolívar tener un relativo respiro durante los tiempos más cruentos de la lucha independentista.

Durante largos meses en Jamaica sufrió el suplicio de una inactividad forzosa, si se descuenta la preparación de diversos artículos y memoriales. En vano apelaba el Libertador al gobernador local, en vano escribía a los ministros en Londres solicitando ayuda militar para los patriotas y exponiendo las ventajas comerciales que podría obtener Inglaterra en las colonias liberadas del yugo español. Sus gestiones tuvieron poco éxito, como las que había realizado en su tiempo Miranda para obtener el apoyo británico. ${ }^{13}$

En aquellas tierras del Caribe británico, Bolívar va a encontrar al fin el respaldo estratégico necesario para reiniciar la lucha por la independencia americana. A respecto, se afirma

13 Lavretski, op. cit., p. 70. 
Carente de apoyo y sin un céntimo en el bolsillo, Bolívar no hallaba posibilidades de ocuparse en algo que pudiera proporcionarle sustento. Pero siempre hay gente generosa en el mundo, y Bolívar conoció, por fin, tales personas también en Jamaica. Maxwell Hyslop que poseía plantaciones en la isla simpatizaba con los patriotas; Julia Cobier, una criolla haitiana muy relacionada con los medios locales y Luis Brion, un hombre de nacionalidad indefinida, rico comerciante y naviero que apoyaba la causa de la independencia de las colonias españolas, le tendieron su generosa mano al Libertador. Carlos Marx apreció altamente las dotes militares de Brion, según palabras de Marx, salió de Londres a Cartagena en una corbeta de veinticuatro cañones equipada en su mayor parte por sus propios recursos, llevando, catorce mil armas y una gran cantidad de equipo militar. Lamentablemente, Brion llegó demasiado tarde, cuando los españoles ya se habían apoderado de Cartagena lo que lo obligó también a refugiarse en Jamaica.
Los nuevos amigos hicieron volver a Bolívar el optimismo y la fe en sí mismo. Brion, quien tenía además una flotilla de barcos pequeños pero veloces no estaba en contra, inclusive, de participar en una expedición al continente a condición de que Bolívar pudiera encontrar suficiente cantidad de hombres arrojados, capaces de lanzar su reto al poderoso Morillo. Por lo pronto, Brion aceptó trasladar a Bolívar en uno de sus navíos a Cartagena, donde Castillo había sido destituido y los patriotas pedían al caraqueño encabezar la defensa de la ciudad asediada.

Bolívar abandonó gustoso Jamaica. Pero en el trayecto llegó a saber que la resistencia de los cercados había sido quebrada y la fortaleza ocupada por los españoles. ¿Qué hacer? ¿Regresar a Jamaica donde estaría como una ratonera incapaz de hacer algo en defensa de su patria? ¡No!

- Tomad rumbo a Haití -ordenó Bolívar al capitán del navío.

El capitán obedeció. ${ }^{14}$

$14 \quad$ Ibid., pp. 71 y 72 . 
Así, al término de aquel año de 1815 prosiguió su exilio; ahora rumbo a Haití, a donde llegó el 31 de diciembre. En el primer país independiente de América Latina y el Caribe, Bolívar recibió el apoyo del presidente Pétion para organizar la primera y segunda expediciones de los Cayos:

A fines de 1815, Bolívar desembarcó en Los Cayos, un pequeño puerto haitiano en los arrecifes de San Luis. Fue recibido inmediatamente por el presidente Alejandro Pétion, uno de los jefes de la lucha de liberación en La Española y fundador de la Primera República de América Latina, Haití, cuya población estaba constituida totalmente por esclavos negros.Pétion comprendía que el triunfo español sobre los patriotas en el continente representaría una amenaza directa también a la independencia de su patria que lindaba en una misma isla con la colonia española de Santo Domingo. Pétion manifestó su disposición de apoyar a Bolívar con armas y equipos, pero con la condición de que el caraqueño lograra unir en torno suyo a la mayoría de los patriotas en el exilio y aceptara abolir la esclavi- tud en todos los territorios que consiguiera liberar. ${ }^{15}$

Posteriormente, el Gran Libertador desembarcó del buque Diana en Barcelona, procedente de costas haitianas, para así concluir con su segundo exilio y emprender desde los combates de Clarines (9 de enero de 1817) la lucha definitiva por la independencia de las colonias de España en América. "[...] En su segundo destierro en Jamaica (mayo 13-diciembre 19, 1815) y en Haití (diciembre 24, 1815-diciembre 18, 1816), estuvo dedicada a la búsqueda de una salida para reiniciar la guerra en Venezuela. Toda la labor como publicista realizada por él en Jamaica y todas las tareas para armar sus expediciones haitianas -Los Cayos (marzo 31, 1816) Jacmel (diciembre 18, 1816)- estuvieron destinadas a ese fin". ${ }^{16}$ En ese año es también un momento clave para el desarrollo de las fuerzas independentistas. El general José de San Martín cruza los Andes para lograr la independencia de la Capitanía General de Chile, entrando triunfalmente en Santiago entre el 12 y el 13 de febrero. Dos años después, el 15 de febrero de 1819 se instala el Congreso de Angostura, y el 7 de agosto se desarrolla la batalla de Boyacá, donde el ejército español

$15 \quad$ Ibid., p. 72.

16 Lovera de-Sola, op. cit., p. 43. 
se rinde y el 10 de agosto el Libertador entra triunfante a Bogotá.

Con esta gran victoria política y militar, Bolívar propuso la formación de la República de Colombia, decretándolo el Congreso el 17 de diciembre de 1819. En ese momento los actuales territorios de los Estados de Colombia, Ecuador, Panamá y Venezuela estaban en gran parte ocupados por las tropas españolas. Dos años después, con la Batalla de Carabobo, el 24 de junio de 1821se generó la destrucción de dos terceras partes del ejército realista. Casi paralelamente en la Nueva España se establece el Plan de Iguala de las Tres Garantías, firmado el 24 de febrero de 1821, fecha en que las fuerzas insurgentes encabezadas por Vicente Guerrero y las realistas de Agustín de Iturbide pactan la independencia de México.

En tanto que la Independencia de Perú es proclamada en Lima por el general José de San Martín el 28 de julio, en la Capitanía General de Guatemala, el 15 de septiembre, se firma el acta de independencia centroamerica, teniendo como uno de sus principales redactores a José Cecilio del Valle. Asimismo en Santo Domingo se declara la independencia del gobierno español y se hace manifiesta la intención de incorporarse a la República de la Gran Colombia el
30 de noviembre. Incluso, en el marco de esa coyuntura, el 7 de septiembre de 1822, Brasil se declara independiente y se forma el Imperio de Pedro I. Para 1824 con las batallas de Junín (6 de agosto) y Ayacucho (9 de diciembre), en que triunfan Bolívar y Sucre, culmina la dominación colonial española en las tierras continentales. Dos años después, el 22 de junio de 1826, se llevó a cabo la propuesta de Bolivar de instalar el Congreso Anfictiónico de Panamá, con representantes de México, Centroamérica, Colombia y Perú, así como con observadores del imperio británico, Estados Unidos y otras potencias. Así, tras una serie de incidentes y conflictos, fue nombrado presidente de la Gran Colombia, y tratando de mantener la unidad de su país y nuestra América, lo que representó una ardua lucha que culminó con la independencia de Bolivia, Colombia, Ecuador, Panamá, Perú y Venezuela.

Su deseo era que Hispanoamérica fuera una gran confederación de estados libres. Finalmente el Libertador, agravada por su salud, falleció el 17 de diciembre de 1830, cerca de Santa Martha, en la hacienda de San Pedro Alejandrino. 